\title{
Radiofrequenzablation erhöht Inzidenz von hitzeinduzierter Thrombose
}

Chait J et al. Radiofrequency Ablation Increases the Incidence of Endothermal Heat-Induced Thrombosis. Ann Vasc Surg 2020; 62: 263-267

Die chronische venöse Insuffizienz ist die häufigste Gefäßerkrankung in den Vereinigten Staaten und betrifft geschätzt bis zu einem Viertel der Bevölkerung mit einer finanziellen Belastung von mehr als einer Milliarde US-Dollar. Die endovenöse Thermoablation ist mittlerweile die Prozedur der Wahl bei der Behandlung der Refluxerkrankung der oberflächlichen Venen. Aktuell besteht ein ganzes Armamentarium unter- schiedlicher Devices und Techniken für die Behandlung dieser Krankheitsentität. Allerdings liegen nicht genügend Daten vor, die die Sicherheit dieser Produkte miteinander vergleichen. Die gefürchtetste Komplikation infolge der Ablation ist aufgrund des Risikos einer Progression zur tiefen Venenthrombose die endothermale hitzebedingte Thrombose (EHIT).
Autoren um Chait aus New York verglichen in ihrer retrospektiven Single-Center-Studie die Inzidenz der EHIT zwischen Radiofrequenzablation (RFA) und endovenöser Lasertherapie (EVLT). Es wurden 3218 aufeinanderfolgende Patienten eingeschlossen, die 10029 endovenösen Ablationen im Bereich der V. saphena unterzogen wurden (66,2\% waren weiblich, Durchschnittsalter 61,9 Jahre). 


\section{Ergebnisse}

3983 EVLT- und 6091 RFA-Prozeduren wurden durchgeführt. Das am häufigsten behandelte Gefäß war die V. saphena magna $(63,6 \%)$, gefolgt von der $V$. saphena parva (25,6\%), akzessorischen Venen $(6,1 \%)$ und Perforatoren (4,6\%). Es kam zu 186 Fällen von EHIT, mit 137 Fällen entsprechend Kabnick-Klassifikation Typ 1 (73,6\%). Die endovenöse Ablation mittels FFA resultierte in signifikant mehr Fällen von EHIT als bei EVLT (109 versus 77; $\mathrm{p}=0,034 ;$ OR 1,52); dieses Ergebnis wurde durch die multivariate Analyse bestätigt.

\section{FAZIT}

In der bislang größten Single-CenterStudie zur Untersuchung der endothermalen Ablation zeigte sich bei der RFA ein höheres Risiko für die Ausbildung einer EHIT als bei der EVLT. Insgesamt war aber die Inzidenz der EHIT relativ niedrig. Obwohl einige Untersuchungen unabhängige
Risikofaktoren für die Entwicklung einer EHIT postulieren, bestehen in der aktuellen Literatur Kontroversen. Die Autoren fordern daher prospektive kontrollierte Studien, um die sicherste, effektivste und wirtschaftlichste Methode für die endovenöse thermale Ablation zu ermitteln.

MOR Dr. med. Benedikt Lampl, Regensburg 